\title{
269 UTILIZING SERUM PROTEOME TO UNDERSTAND RESPONSE AND RESISTANCE TO IMMUNE CHECKPOINT INHIBITORS IN ADVANCED NON-SMALL CELL LUNG CANCER
}

${ }^{1}$ Leeseul Kim*, ${ }^{2}$ Young Kwang Chae, ${ }^{2}$ Dong-Uk Lee. ${ }^{1}$ AMITA Health Saint Francis Hospital Evanston, Chicago, IL, USA; ${ }^{2}$ Developmental Therapeutics Northwestern Medicine, Chicago, IL, USA

Background Predictive biomarkers are more in need considering that responses and resistance to immune checkpoint inhibition (ICI) are poorly understood. We used a validated serumbased proteomic test, Primary Immune Response (PIR), to predict response and Protein Set Enrichment Analysis (PSEA) scores to elucidate mechanisms of early resistance to ICI in patients with advanced non-small cell lung cancer (NSCLC). (Muller et al., 2020)

Methods Serum of 43 consented NSCLC patients was collected prospectively at 2 timepoints: baseline (prior to ICI initiation) and 3 weeks after ICI initiation (median 22 days). Blinded samples were classified by the PIR test. Clinical response was evaluated using RECIST. Outcomes, including progression-free survival (PFS) and overall survival (OS), were analyzed by PIR classifications as intermediate/sensitive (not resistant) vs. resistant at baseline and 3 weeks. Multivariable regression was performed. PSEA scores indicating activity of 10 processes of interest (e.g., Type 1 immunity (Th1), complement, interferon-gamma (IFN $\gamma)$ ) were compared between PIR groups.

Results Of the 43 patients, 28 received chemotherapy with ICI (chemo+ICI) and 15 received ICI alone. 31 of 43 patients (72\%) were treatment naïve at baseline blood draw. PIR-resistant patients had worse survival compared to patients classified as not resistant (HR, 10.4; 95\%CI, 1.3-81; $\mathrm{P}=0.025)$. OS was also significantly lower for patients with PIR resistant result at 3 weeks (HR, 9.1; 95\%CI, 1.2-72; $\mathrm{P}=0.036)$. The difference in survival between PIR classification groups was consistently observed in the treatment naive patients treated with chemo+ICI $(\log \operatorname{rank} P=0.02)$. No significant differences were observed in PFS. Clinical and pathologic characteristics, including PD-L1 expression, were not significantly associated with PIR result. In multivariable analysis including performance status, line of therapy, and PD-L1 status, PIR resistant remained a significant negative prognostic factor (HR, 8.2; 95\%CI, 1.01-67; P = 0.049). PSEA scores at baseline and 3 weeks after ICI initiation showed significantly higher levels of complement, IFN $\gamma$, Th1, immune tolerance, and a lower level of wound healing (all $\mathrm{P}<0.0001$ ) in PIR-resistant vs. PIR-intermediate/sensitive.

Conclusions These data further validate the utility of the PIR test in predicting patient survival on ICI. Processes associated with PIR resistant result included activation of complement, IFN $\gamma$, Th1, and immune tolerance, elucidating early mechanisms of resistance to ICI in a clinical cohort.

http://dx.doi.org/10.1136/jitc-2021-SITC2021.269 